\title{
Elbe 2020 - \\ Investigating a River-Sea System from Upstream into the North Sea
}

H. Brix, N. Kamjunke, I. Bussmann, E. Achterberg, P. Dietrich, P. Fischer, G. Flöser, M. Friedrich, F. Geißler, U. Koedel,

M. Koschorrek, L. Rewrie, C. Schütze 


\section{The Setting}

- Understanding river-sea-systems requires a thorough understanding of processes that span different Earth system compartments

- Issues when scientific disciplines interact. Differences in:

- Measurement and calibration standards

- Quality Control Approaches

- Data Formats and Handling

- MOSES (Modular Observation Solutions for Earth Systems) addresses this type of problem through:

- Sensor comparison and intercalibration campaigns

- Joint field campaigns involving partners from different Earth compartment and disciplines 


\section{Objectives}

- Establish scientifically sound and resilient sampling procedures for multiple ships (inland waters and ocean going, fresh and sea water, ...)

- Create reference data for main environmental parameters for future investigations of extreme events

- Test communication logistics

- Establish common data flows and protocols 


\section{Logistics and Realization}

- Four research vessels from four research institutions

- Duration: two months

- Measurements:

- Standard hydrological and oceanographic parameters

- Nutrient and carbonate systems

- Two cruises in the river (fresh water and tidal sections)

- Three ships in the German Bight

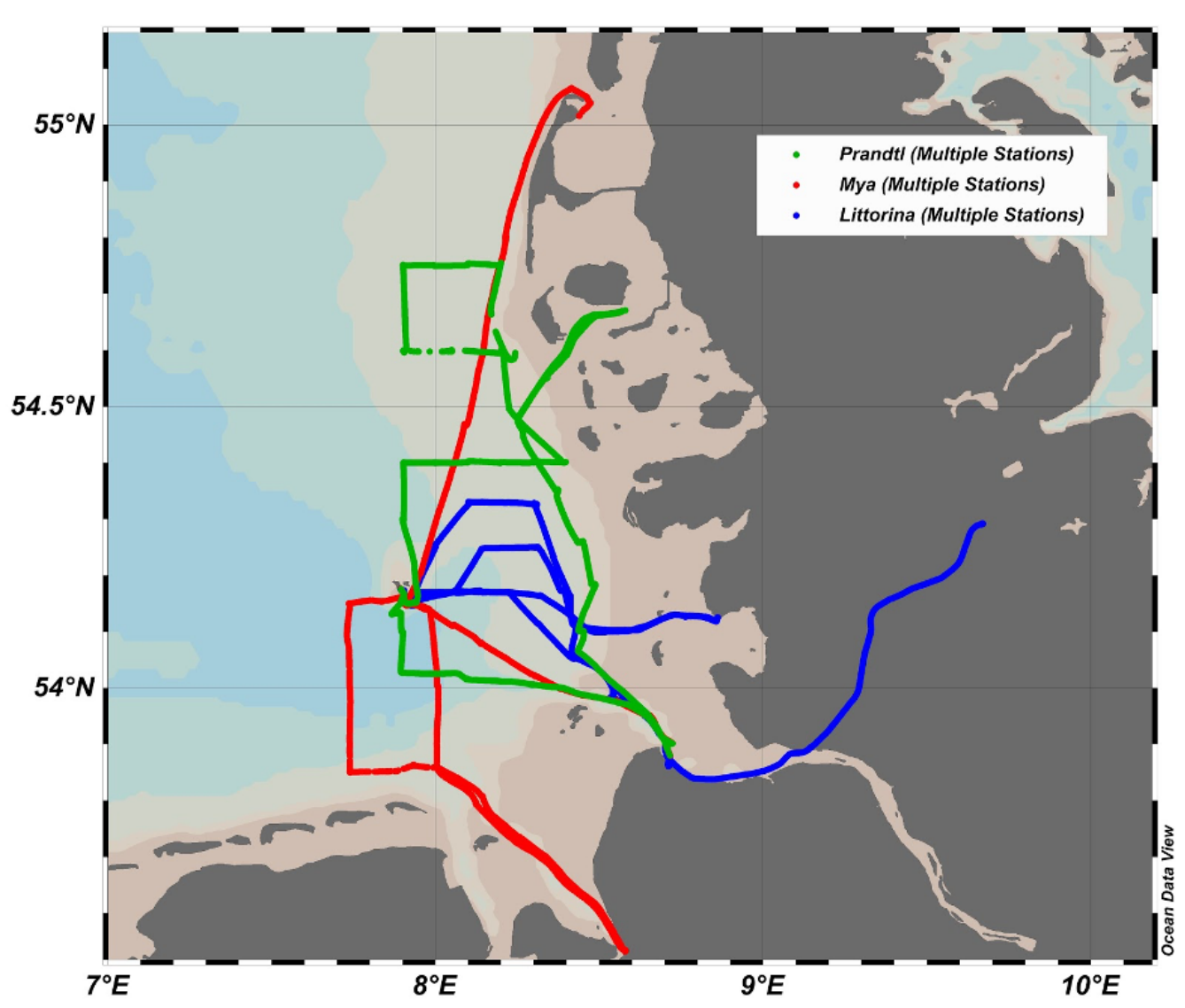




\section{Results along the river: Helgoland - Czech Republic}

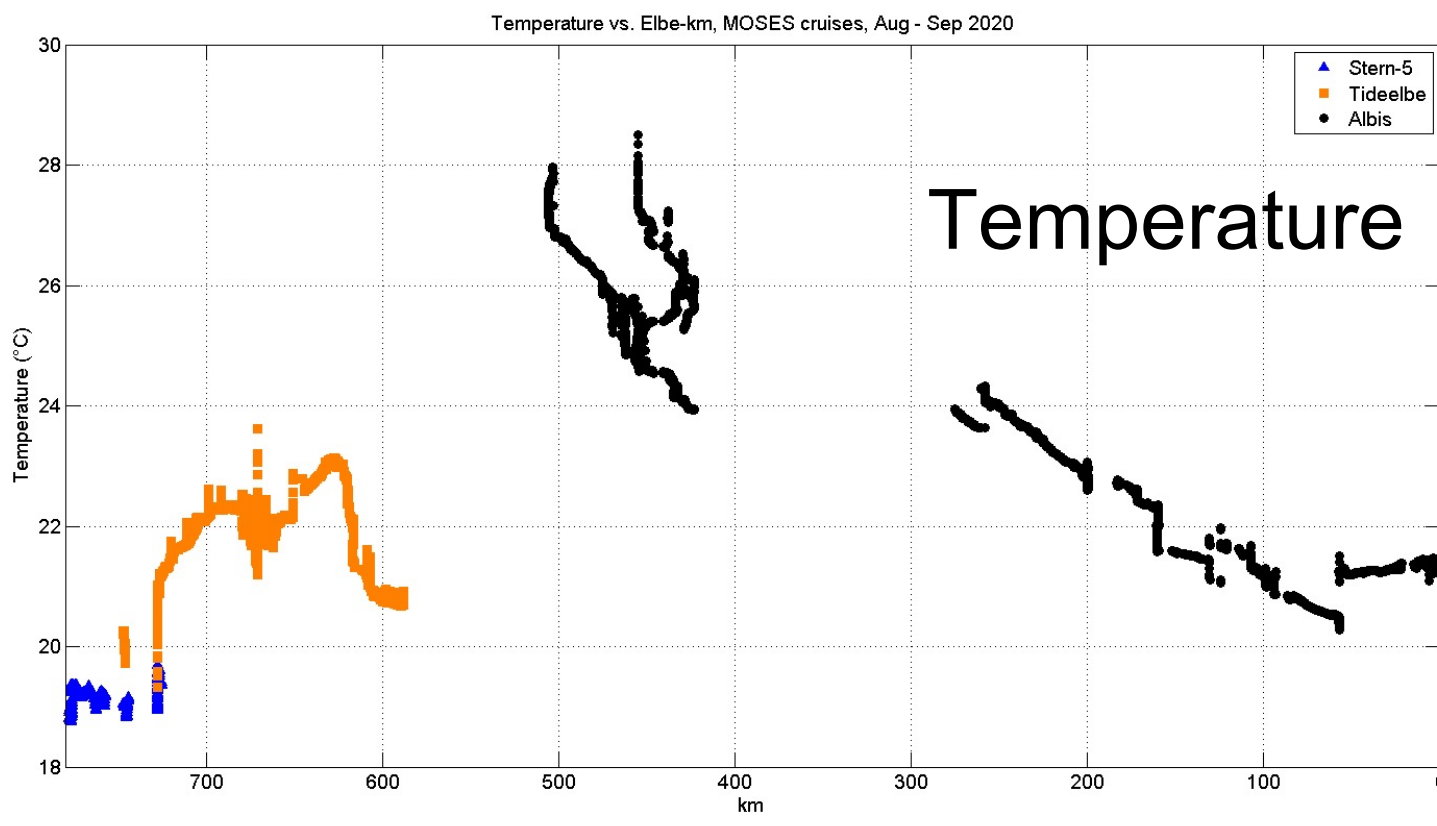

German

Bight

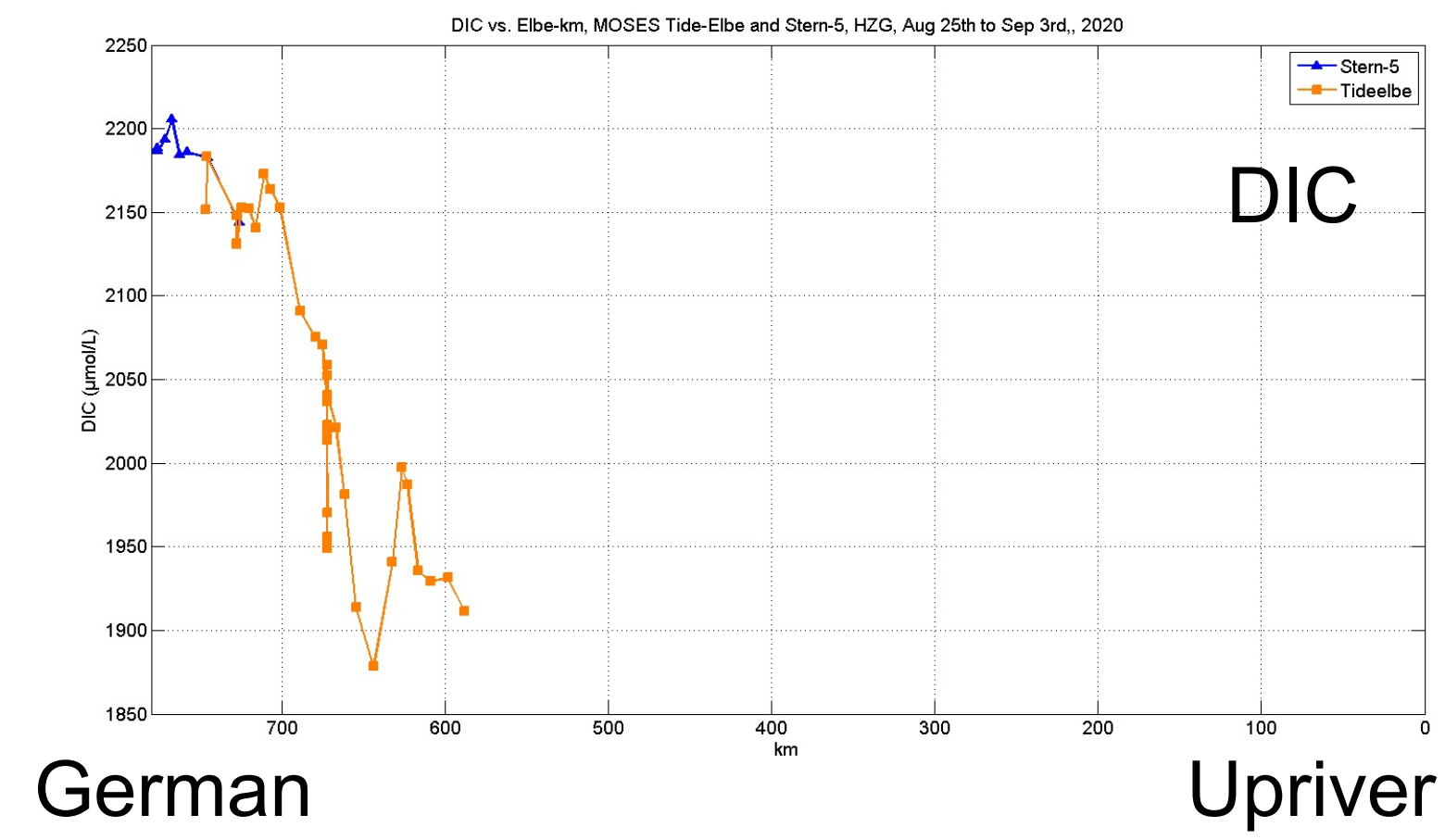

Bight

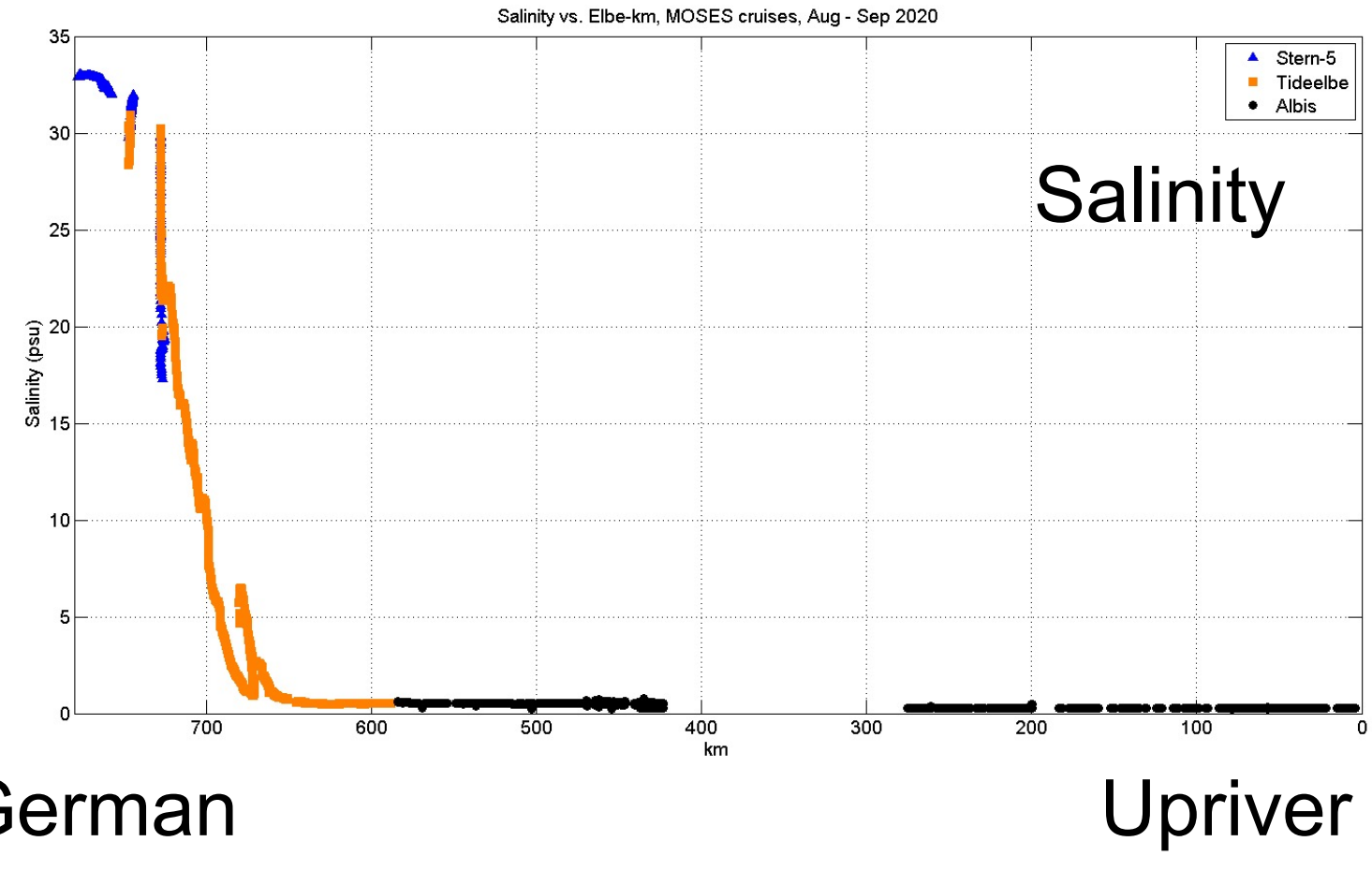

Bight

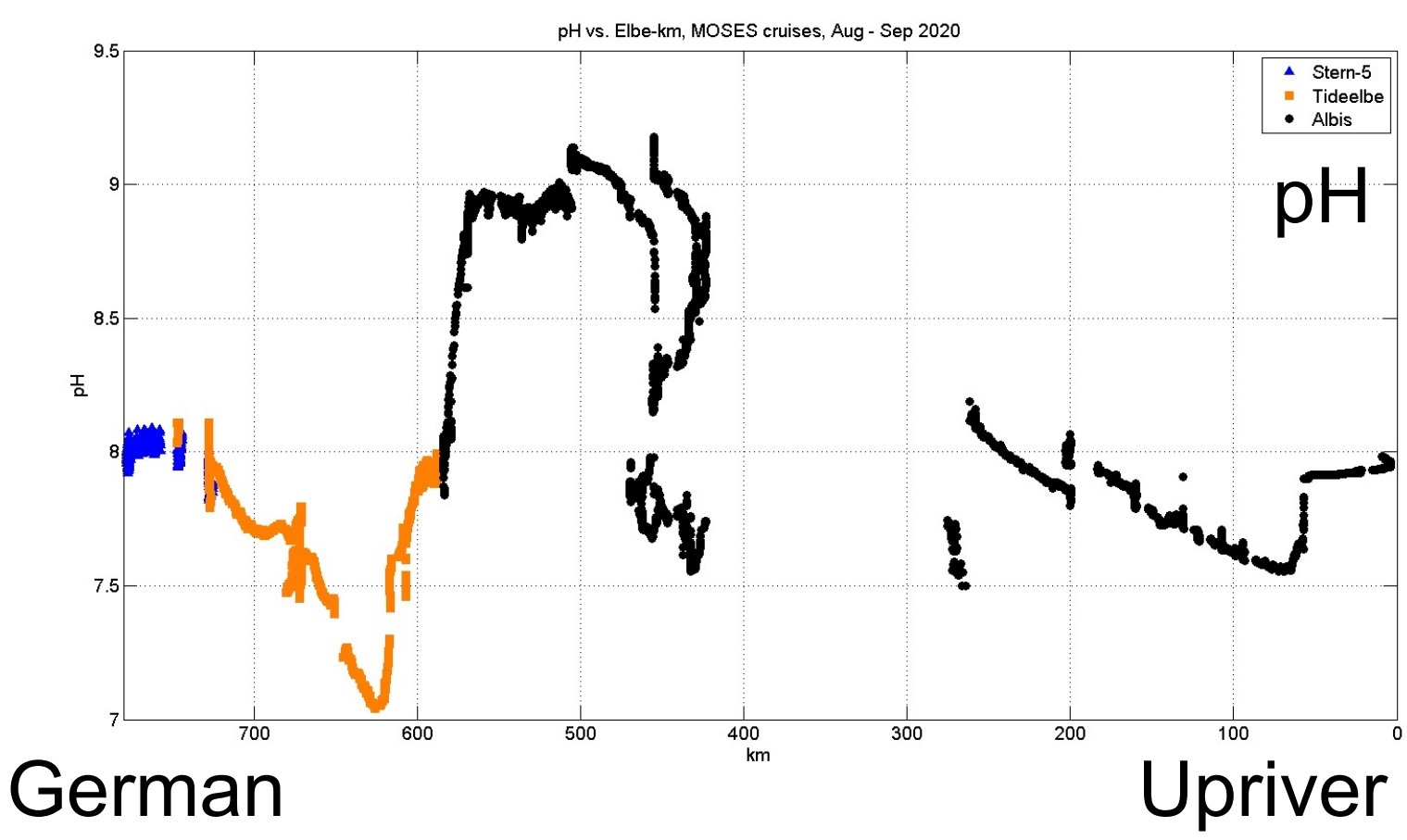

Bight 


\section{Results along the river:}

\section{Helgoland - Czech Republic}
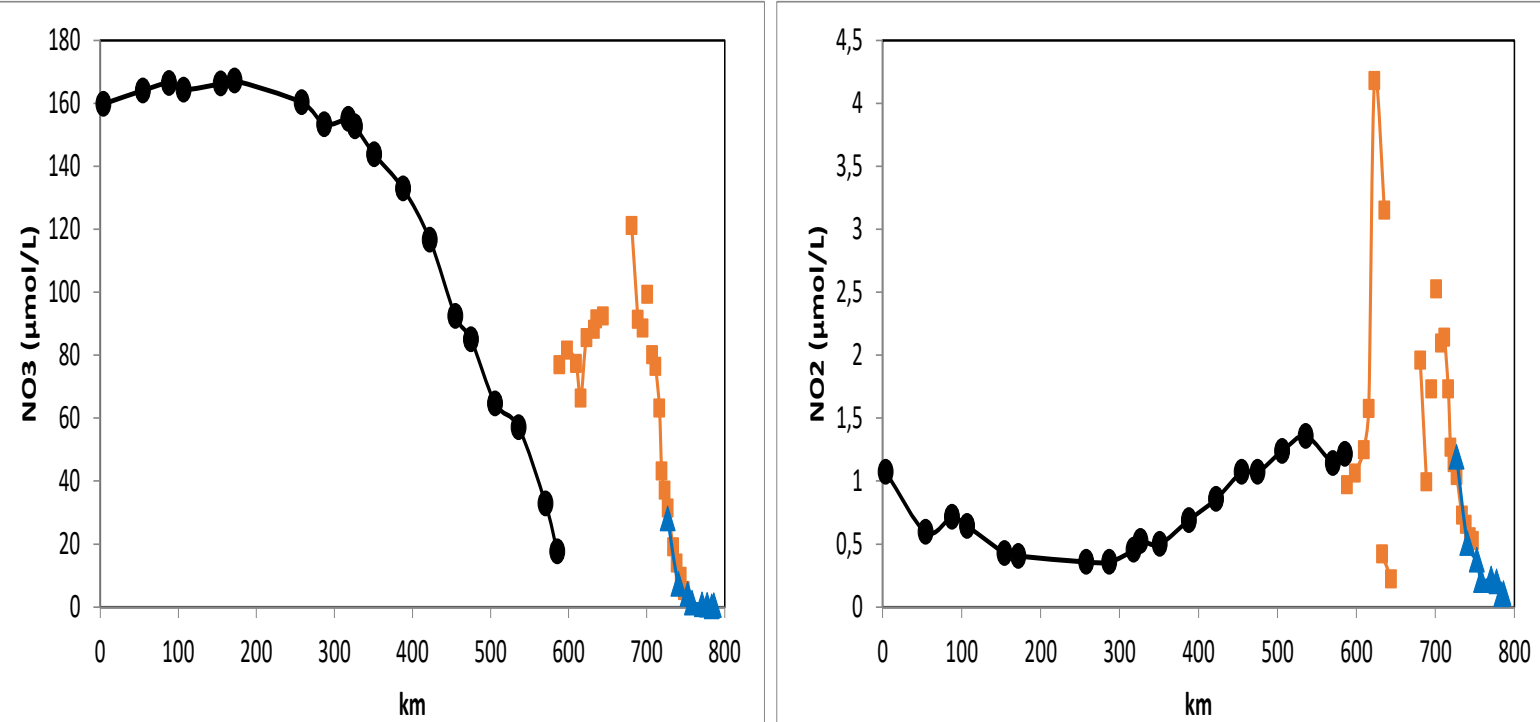

Modular Observation Solutions for Earth Systems
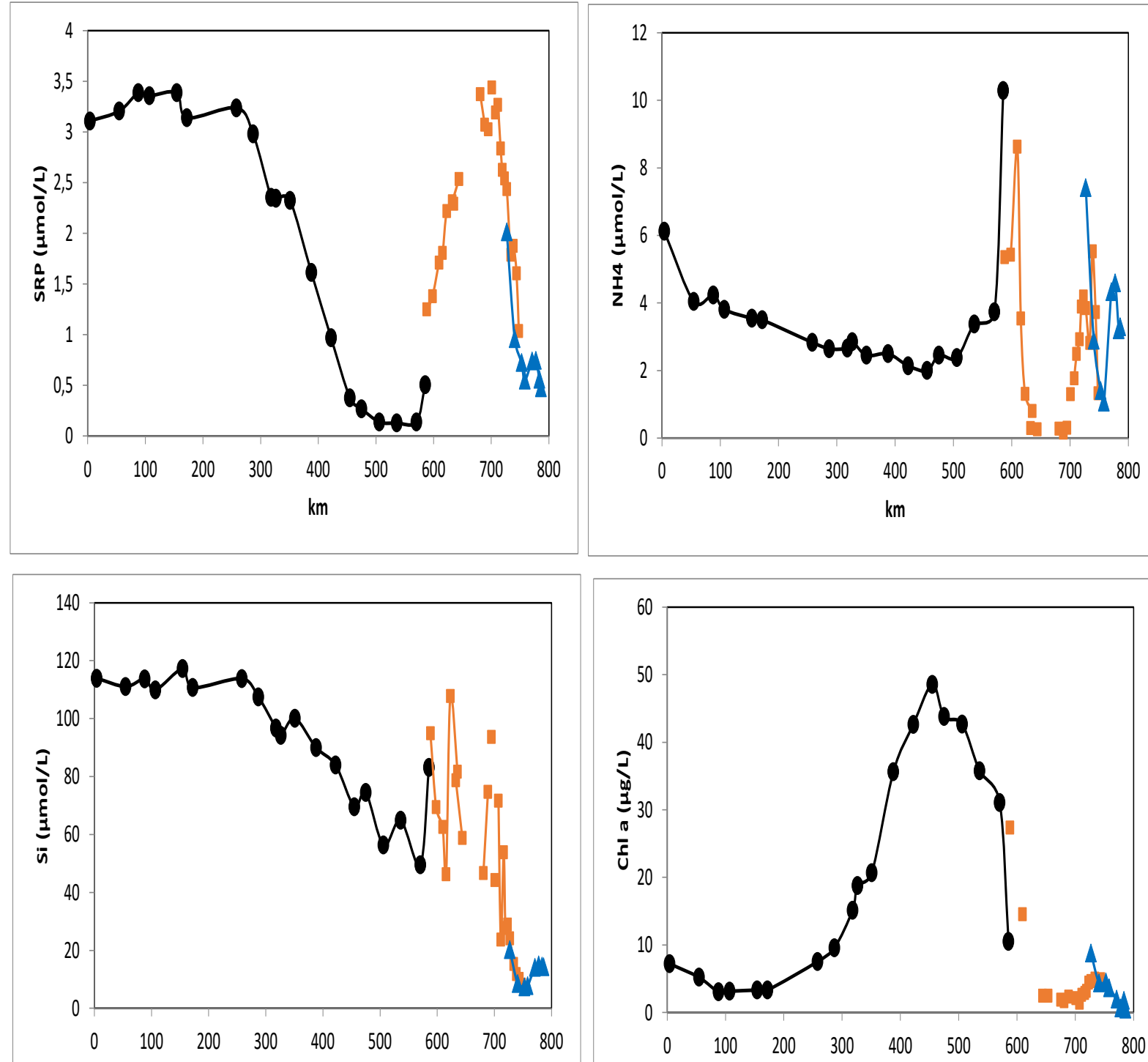


\section{Results for the Tidal Cycle}
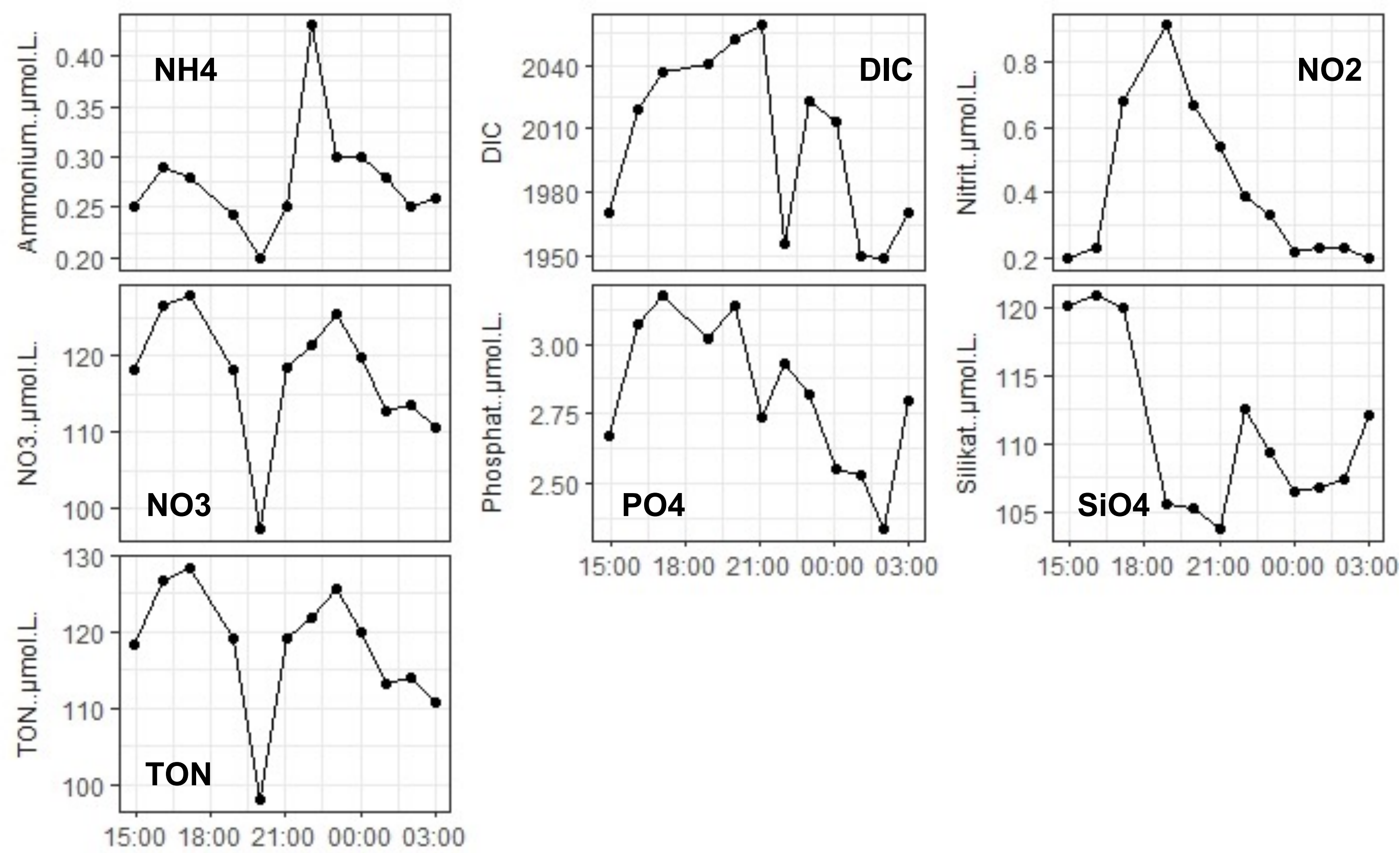


\section{Results in the estuary and beyond}
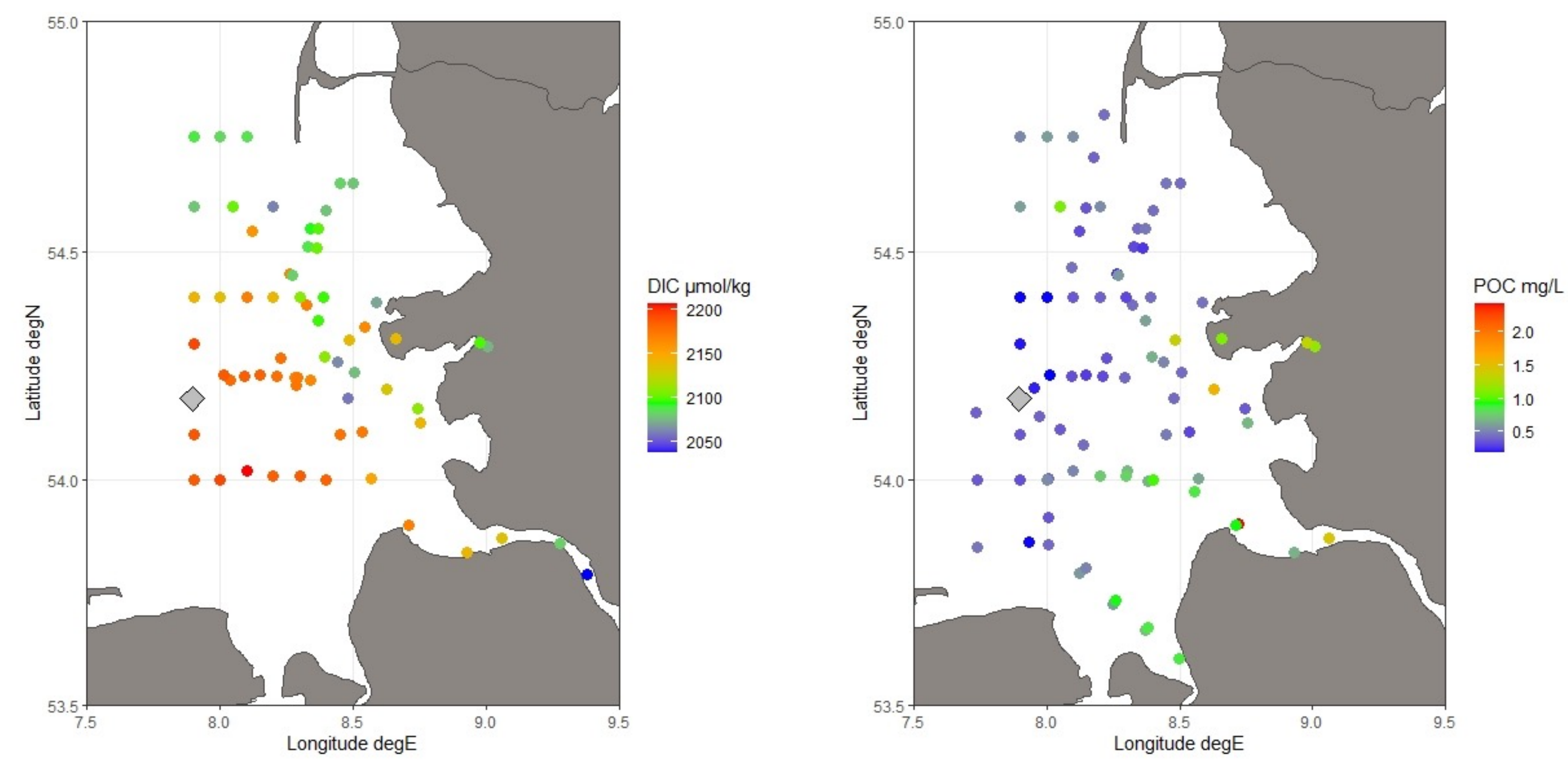


\section{Conclusions}

- Measurements across compartments require logistical effort and challenge old habits

- Cross-calibration, shared data handling and logistics are required

- Results help answer local / regional research question as well as enhance systems understanding

- Challenges:

- Communication in the field

- Sustaining network for operations to monitor extreme events 


\section{Acknowledgements}

- Crew and captian of RVs Albis, Littorina, Ludwig Prandtl and Mya II

- Numerous lab technicians at all participating institutes

- Numerous colleagues "behind the scenes" supporting with logistics, instruments, knowhow and advice

Thank you for your attention! 